Please do not remove this page

RMIT

UNIVERSITY

\title{
Optimum sensitive area of surface acoustic wave resonator chemical and bio-sensors
}

Kalantar-Zadeh, Kourosh; Powell, David; Wlodarski, Wojtek

https://researchrepository.rmit.edu.au/esploro/outputs/9921859986101341/filesAndLinks?institution=61RMIT_INST\&index=null

Kalantar-Zadeh, K., Powell, D., \& Wlodarski, W. (2005). Optimum sensitive area of surface acoustic wave resonator chemical and bio-sensors. Proceedings of the IEEE Sensors 2005 Conference, 1229-1232.

https://doi.org/10.1109/ICSENS.2005.1597928

Published Version: https://doi.org/10.1109/ICSENS.2005.1597928

Repository homepage: https://researchrepository.rmit.edu.au

(c) 2005 IEEE. Personal use of this material is permitted. However, permission to reprint/republish this material for advertising or promotional purposes or for creating new collective works for resale or redistribution to servers or lists, or to reuse any copyrighted component of this work in other works must be obtained from the IEEE.

Downloaded On 2023/04/26 23:32:08 +1000 


\title{
Optimum Sensitive Area of Surface Acoustic Wave Resonator Chemical and Bio-Sensors
}

\author{
David A. Powell, Kourosh Kalantar-zadeh, and Wojtek Wlodarski \\ Sensor Technology Group, School of Electrical and Computer Engineering \\ RMIT University City Campus, Melbourne, VIC 3001, AUSTRALIA \\ email: david.powell@ieee.org
}

\begin{abstract}
A model is developed to map the variation of sensitivity of a Surface Acoustic Wave (SAW) resonator sensor over its surface, in order to find the region with maximum sensitivity. The model is based on a combined Coupling of Modes (COM) and periodic Green's function analysis. In order to extend the analysis to layered media, a new efficient technique is introduced to account for the mechanical interactions with buried electrodes. Using this technique the sensitivity calculations are found to be in good agreement with measurements. It is also shown that whilst changes in other parameters influence the sensitivity, it is the velocity change which most strongly determines the overall frequency change.
\end{abstract}

\section{INTRODUCTION}

SAW sensors based on shear-horizontal ( $\mathrm{SH}$ ) waves are well established devices for studying the properties of liquids, and chemical or biological reactions occurring within a liquid environment. Compared to SAW devices based on a delay-line configuration, resonator structures offer higher frequency stability for a given device size, due to the multiple reflections increasing the group delay. Figure 1 shows a schematic representation of a 2-port resonator SAW structure (not to scale). Regions B-C and D-E represent the input and output interdigital transducers which are connected to the external circuit. Regions A-B and E-F are short-circuited electrode gratings of the same period as the IDTs. These gratings serve to reflect acoustic energy and create a resonant cavity. Region C-D is a grating with a different period to that of the IDTs. Its purpose is to trap the SH wave towards the surface without causing significant reflection.

SAW transducer sensitivity has been investigated by both theoretical and experimental means (e.g. [1]). However, in most cases it is assumed that the device interaction with the analyte is uniform over the surface. In applications such as bio-sensing, this assumption is not well grounded. Using such a model it is not possible to conclude which part of the device is most

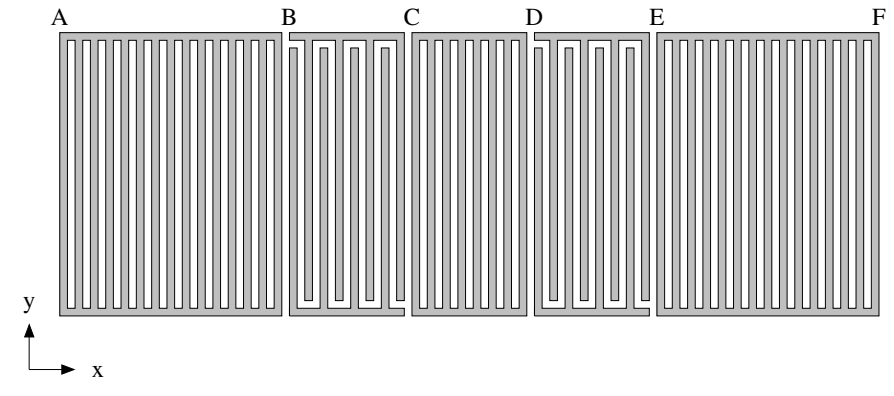

Fig. 1. Layout of 2-port resonator SAW sensor in $(x, y)$-plane

sensitive to surface perturbations. It has previously been shown that for SAW delay lines with electrodes exposed to the analyte, sensitivity varies significantly over the device surface [2]. For resonator type SAW devices even stronger sensitivity variation is expected, since acoustic energy decays exponentially into the reflective gratings. Thus it is necessary to investigate the effect of inhomogeneous mass-loading, in order to find the region of the SAW device where sensitivity is highest.

This is particularly important in cases where only a small quantity of selective material is available, so that it can be placed on the most sensitive region of the SAW device. Similar considerations also apply when a limited amount of analyte is available.

\section{Measurements}

The 2-port resonator sensors were fabricated on a $36^{\circ}-\mathrm{YX} \mathrm{LiTaO}_{3}$ substrate. Gold electrodes of $0.1 \mu \mathrm{m}$ thickness were patterned, and the period of the IDT electrodes was $20 \mu \mathrm{m}$. A small spacing adjustment is made at point $\mathrm{D}$ to ensure a single resonant frequency with high quality factor (Q). A $0.4 \mu \mathrm{m}$ layer of $\mathrm{SiO}_{2}$ was deposited on the device surface using RF magnetron sputtering, in order to protect the electrodes. The resultant devices had resonant frequencies in the range of $205-206 \mathrm{MHz}$.

Sensitivity measurements were performed by placing the SAW devices within the oscillator circuit before and 
after the addition of a perturbing mass, and measuring the change in oscillation frequency. The system was designed to ensure that the pressure placed on the SAW device by measurement probes was repeatable. The added mass was AZ1512E photo-resist, of thickness $0.6 \mu \mathrm{m}$. This relatively thick layer was chosen to ensure that a strong response was observed. Because the photoresist is a soft material the response should be mainly attributable to its mass, although some viscosity influence would also be expected. Each measurement was repeated simultaneously on 5 different devices, which were fabricated in the same batch.

Figure 2 shows the results of the measurement, along with calculated values which will be discussed in Section III. The horizontal axis of the graph gives the location along the $x$-axis as shown in Fig. 1, with the corresponding points $\mathrm{A}$ through $\mathrm{F}$ being marked out. Each circle marked on the graph represents a simulated mass sensitivity value. The corresponding horizontal line represents the region over which the additional mass has been deposited or modelled. The nearby crosses represent the measured values of sensitivity. In all cases the mass has been deposited over the complete width of the device in the $y$-direction.

\section{MODEL}

The model is based on the formulation of a spectral domain Green's function $\bar{G}\left(s_{1}\right)$, which describes the acoustic wave properties of the substrate and guiding layer. Since the devices have a large number of electrodes, a periodic Green's function $G_{p}(x)$ is used to reduce computation time. Using this function, the harmonic admittance of an infinite electrode array can be calculated using the Boundary Element Method (BEM). A fitting procedure [3] is used to derive Coupling of Modes (COM) parameters from this admittance curve. The COM model has parameters for propagation velocity $v$, reflection $\kappa$, transduction $\alpha$, capacitance $C$ and attenuation $\gamma$.

This approach is advantageous because it combines the speed and simplicity of the COM model with the accuracy of the rigourous BEM solution. The application of this model to layered SAW resonators operating in liquid media was previously reported by the authors [4].

The COM parameters are used to derive a P-matrix for each electrode pair in the device, and this P-matrix can be calculated for the cases with and without massloading, thus allowing mass sensitivity to be calculated along the length $(x)$ of the device. Variation of the mass sensitivity across the width $(y)$ of the device has been neglected in this model. This effect can be assumed to be insignificant since the device geometry is constant across its width, which is much greater than the acoustic wavelength.

Using the P-matrix model, the complete 2-port parameters of the SAW device can be calculated and the resonant frequency determined. The shift in this resonant frequency, divided by the mass which causes the shift is considered as a measure for the device sensitivity. The model shows how a local change in mass loading at a region on the device surface affects the resonant frequency of the device.

\section{A. Mechanically Thin Electrodes}

Provided the electrodes are of negligible thickness compared with the acoustic wavelength, and are made from a light material, their mechanical properties do not need to be taken into account and they can be considered as being infinitely thin. This assumption greatly simplifies the analysis, and speeds up the computation. Figure 2 shows the theoretical and measured sensitivity of our test device based on this assumption. It can be seen that, as expected, the sensitivity is greatest at the centre of the device. The region B-E will be denoted the optimum region, although this region is arbitrarily selected, and may be chosen differently for another device geometry. This region accounts for the majority of the total device response.

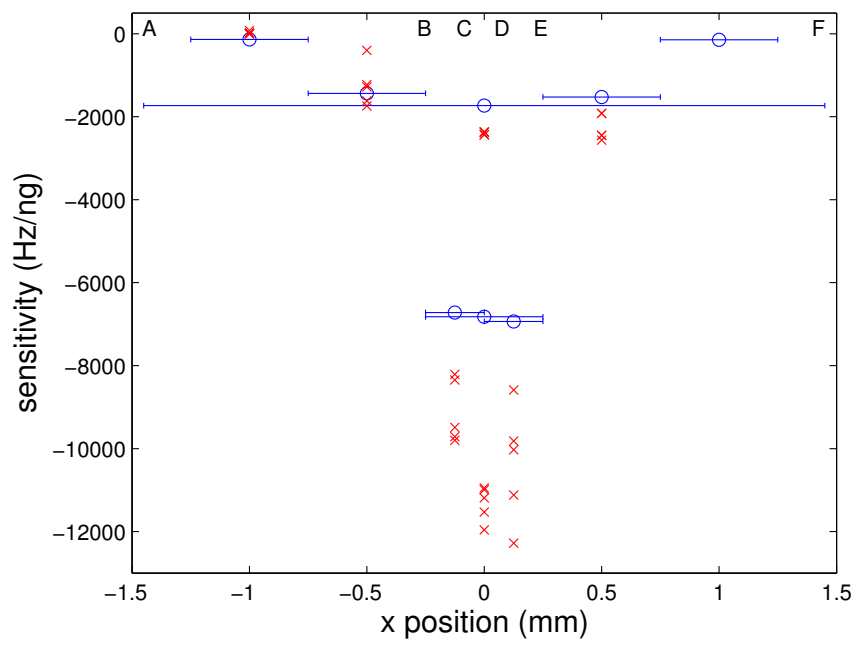

Fig. 2. Sensitivity with thin electrodes

The agreement in shape is quite good, however the measured sensitivity is more confined to the centre than the model predicts, and the average sensitivity over the complete device is also greater. This disagreement can be 
attributed to the relatively heavy electrodes which were not included in the model. The heavy electrodes enhance the acoustic reflection, thus confining the acoustic energy to the centre of the device. They also trap acoustic energy to the surface, thus further increasing the average mass sensitivity of the device.

\section{B. Mechanically Thick Electrodes}

The results of Section III-A indicate that mechanical electrode interactions have a significant influence on the performance of our device. Typically the Finite Element Method (FEM) is used to account for such interactions, however FEM becomes cumbersome in the case of electrodes embedded within layered media [5]. In this paper we utilise a simpler technique based on the formulation of a matrix eigen-operator [6]. Briefly, the piezoelectric equations are manipulated into the following form:

$$
\left[\begin{array}{cc}
\mathcal{L}_{11}(x) & \mathcal{L}_{12}(x) \\
\mathcal{L}_{21}(x) & \mathcal{L}_{22}(x)
\end{array}\right] \boldsymbol{\tau}=\frac{\partial}{\partial z} \boldsymbol{\tau}
$$

where $\boldsymbol{\tau}=\left[\begin{array}{llll}\boldsymbol{u} & \varphi & \boldsymbol{T} & D_{z}\end{array}\right]^{T}$ are the mechanical displacement, voltage, $z$-components of stress and $z$ component of electric flux respectively. The device configuration to be modelled is shown in Fig. 3.

\begin{tabular}{l|c|c|c|c}
\hline (i) & \multicolumn{4}{c}{ Guiding Layer } \\
\hline (ii) & Electrode & Guiding Layer & Electrode & $\mathrm{Z}_{2}$ \\
\hline (iii) & \multicolumn{2}{c}{$\begin{array}{c}\mathrm{z} \\
\text { Substrate }\end{array}$} & $\stackrel{\mathrm{z}=\mathrm{z}_{1}}{\longrightarrow}$ &
\end{tabular}

Fig. 3. Cross-section of electrode configuration in $(x, z)$ plane

Regions (i) and (iii) are accounted for using the existing Green's function, and for simplicity it is assumed that the charge is located only at $z=z_{1}$. The new model is required for region (ii), where there are 2 different sets of material constants, alternating periodically. To allow a numerical solution of (1), the material constants (or their combinations which appear in $\mathcal{L}$ ) are represented by a truncated Fourier series. For example, density $\rho$ is represented as:

$$
\rho(x)=\sum_{n=-N}^{N} \rho_{n} e^{j 2 \pi n / p x}
$$

The elements of $\tau$ are represented by a Fourier series with an additional phase shift $\beta$ :

$$
\tau_{l}(x, z)=\sum_{m=-N}^{N} \tau_{l, m}(z) e^{j(2 \pi m / p+\beta) x}
$$

By substituting equations (3) and (2) into (1), an algebraic eigenvalue problem results. From the eigenvalues and eigenvectors, a transmission matrix $\Phi$ can be developed, relating the field values at the top and bottom of region (ii) in Fig. 3:

$$
\tau_{m, k}\left(z_{1}\right)=\sum_{n=-N}^{N} \sum_{k=1}^{8} \Phi_{m n k l} \tau_{n, l}\left(z_{2}\right)
$$

This can be regarded as a generalisation of Adler's [7] chain matrix technique for layered media, extended to the case where the material constants are a function of $x$. The new model will be presented in detail elsewhere.

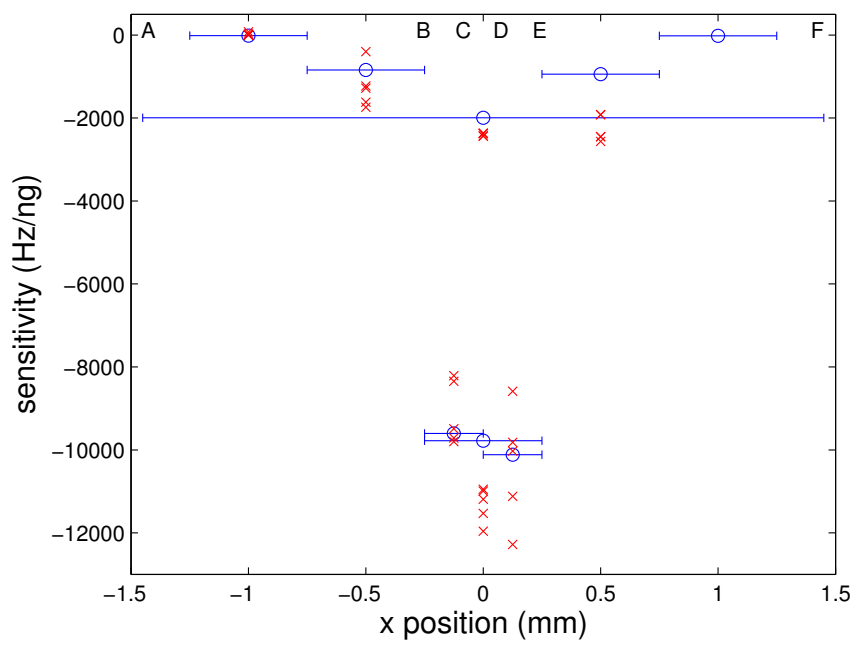

Fig. 4. Sensitivity with thick electrodes

The mechanical electrode model was incorporated into the periodic Green's function analysis, and the sensitivity was calculated as before. The result is shown in Fig. 4. The sensitivity shows better agreement with measurements in the case where complete device is mass loaded, as does the sensitivity in the optimum region. Because the $\mathrm{SiO}_{2}$ film is deposited conformably over a surface covered with electrodes, the top surface of the device has corrugations, which would also need to be included in the model for improved accuracy.

\section{Influence of Other Parameters}

In order to test the relative importance of parameters $\alpha, \kappa, C$ and $\gamma$ in determining sensitivity, the sensitivity was calculated with these parameters held constant, with only $v$ varying. The resulting sensitivity distribution is shown in Fig. 5, being compared with the measured values. It can be seen that the results do not differ greatly from the full simulation, which indicates that in this case the velocity change is predominant. 


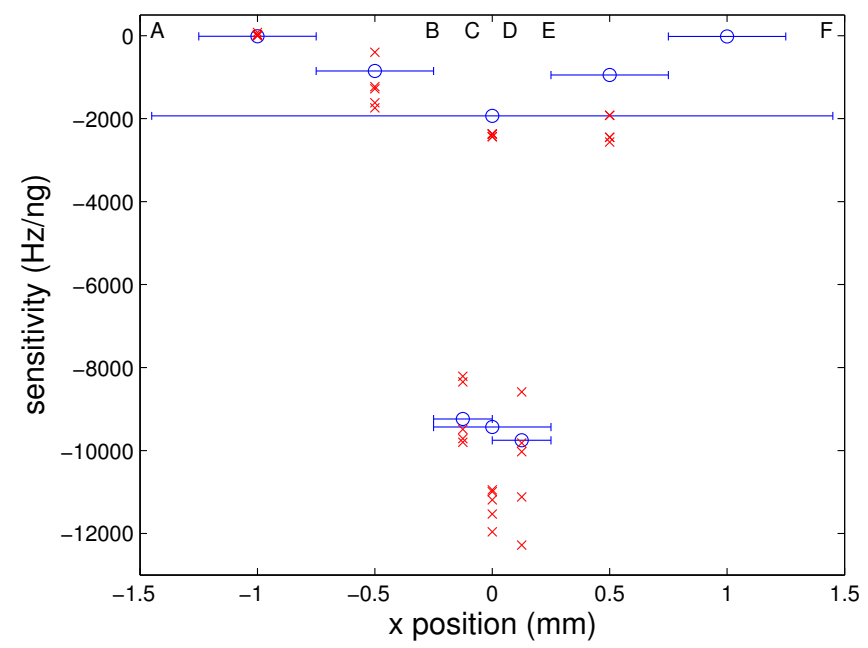

Fig. 5. Sensitivity considering only velocity change

\section{Discussion}

It is of interest to compare the sensitivity of the complete device, the optimum region and of an idealised infinite length IDT. The table below shows a comparison of these sensitivity calculations. To compare with an infinite IDT we must consider mass loading per unit area. This shows that the optimum region accounts for $84 \%$ of the complete device sensitivity. The sensitivity of the device to homogeneous loading is closely related to the sensitivity of the velocity and resonant frequency of an infinite IDT. These parameters differ mostly because of the sensitivity of the reflection coefficient [4].

\begin{tabular}{|c|c|}
\hline Structure & Sensitivity $\mathrm{cm}^{2} / \mathrm{g}$ \\
\hline Infinite IDT $v$ & -96.4 \\
Infinite IDT $f$ & -117.8 \\
Complete Resonator & -99.3 \\
Optimum Region & -83.5 \\
\hline
\end{tabular}

The model suggests that for bio-sensing applications with a limited quantity of available selective material, to achieve maximum response, this material should only be immobilised over the optimum region of the SAW device. It must be stressed that there are other factors which also need to be taken into account including mass transport, optimum receptor concentration and whether a flow-through or static cell has been used [8].

On the other hand, in situations such as environmental gas monitoring, it is more reasonable to assume that there is an unlimited quantity of analyte interacting with the complete device surface. A comparison of delay line and resonator structures has been made under these assumptions, resulting in quite different conclusions [9].
Although mass sensitivity is investigated here the mechanism for varying sensitivity across the device surface is not specific to mass loading. The principal is primarily the means by which the device structure translates a local velocity change $\Delta v / v$ to an overall change in the oscillation frequency of the device $\Delta f / f$. Thus similar results would be expected for sensors based on changes in other parameters such as viscosity or conductivity and for other types of SAW mode.

\section{CONCLUSION}

It was shown that a fixed quantity of analyte will have a greater effect on the device response if it is immobilised or adsorbed onto a small region at the centre of the device. The authors propose to test this conclusions using biological materials in a liquid environment, to quantify the performance change when material is immobilised only over the optimum region of the SAW.

\section{ACKNOWLEDGEMENTS}

The assistance of Professor Ali Reza Baghai-Wadji in developing the model for electrodes buried within layered media is gratefully acknowledged.

\section{REFERENCES}

[1] Z. Wang, J. D. N. Cheeke, and C. K. Jen, "Perturbation method for analyzing mass sensitivity of planar multilayer acoustic sensors," IEEE Trans. Ultrason., Ferroelect., Freq. Contr., vol. 43, no. 5, pp. 844-851, Sept. 1996.

[2] G. L. Harding, "Mass sensitivity of Love-mode acoustic sensors incorporating silicon dioxide and silicon-oxy-fluoride guiding layers," Sens. Act. A: Phys., vol. 88, pp. 20-28, 2001.

[3] J. Koskela, V. P. Plessky, and M. M. Salomaa, "SAW/LSAW COM parameter extraction from computer experiments with harmonic admittance of a periodic array of electrodes," IEEE Trans. Ultrason., Ferroelect., Freq. Contr., vol. 46, no. 4, pp. 806-816, 1999.

[4] D. A. Powell, K. Kalantar-zadeh, and W. Wlodarski, "Comprehensive analysis of SAW sensor performance in liquid media by Green's function method," in Proc. IEEE Ultrason. Symp., Oct. 2003, pp. 146-149.

[5] S. Ballandras, V. Laude, T. Pastureaud, W. Wilm, W. Daniau, A. Reinhardt, and W. Steichen, "A FEA/BEM approach to simulate complex electrode structures devoted to guided elastic wave periodic transducers," in Proc. IEEE Ultrason. Symp., 2002, pp. 309-312.

[6] A. R. Baghai-Wadji, A Unified Approach for Construction of Green's Functions: Theory and Applications (Habilitation manuscript). Vienna: Vienna University of Technology, 1994.

[7] E. L. Adler, "Matrix methods applied to acoustic waves in multilayers," IEEE Trans. Ultrason., Ferroelect., Freq. Contr., vol. 37, no. 6, pp. 485-490, Nov. 1990.

[8] E. Gizeli and C. R. Lowe, Eds., Biomolecular Sensors. London: Taylor \& Francis, 2002.

[9] A. Mauder, "SAW gas sensors: comparison between delay line and two port resonator," Sens. Act. B: Chem., vol. 26-27, pp. 187-90, 1995. 